\title{
Design and Evaluation of Transmitting Antennas for Solar Power Satellite Systems
}

\author{
Abdullah Alogla \\ Department of Electrical Engineering \\ College of Engineering \\ University of Ha'il \\ abdullahogla@gmail.com
}

\author{
Mohamed Abdelaziz Hassan Eleiwa \\ Department of Electrical Engineering \\ College of Engineering \\ University of Ha'il \\ ma.eleiwa@uoh.edu.sa
}

\author{
Hammad Alshortan \\ Department of Electrical Engineering \\ College of Engineering \\ University of Ha'il \\ en.hh2010@hotmail.com
}

\begin{abstract}
This study attempts to identify, design, and evaluate transmitting antennas for Solar Power Satellite (SPS) systems. The design approach aimed at meeting the SPS operational requirements at ISM bands, namely $2.4-2.5 \mathrm{GHz}$ for the NASA and $5.725-5.875 \mathrm{GHz}$ for the JAXA models. The primary attributes of SPS antennas for transmitting Beamed High-Power Microwaves (BHPMs) are high power handling capability, efficiency, and directivity with narrow beamwidth and lower sidelobe levels. Using a planar end-fed $20 \times 20$ SWA module, the whole planar Slotted Waveguide Antenna Arrays (SWAAs) were designed for both the NASA and JAXA reference models having $1 \mathrm{~km}$ diameter antenna aperture, peak power level over $1 \mathrm{GW}$, directivity over 80dBi, Side Lobe Level (SLL) less than $20 \mathrm{~dB}$, and pencil beam with HPBW less than $0.01^{\circ}$. The proposed slotted waveguide transmitting antenna arrays fulfilled the operational requirements for both the NASA and JAXA SPS reference models. Due to the higher operating frequency, the results showed that the proposed planar SWA array performs better on the JAXA than on the NASA SPS model.
\end{abstract}

Keywords-solar power satellites; microwave power transmission; slotted waveguide antenna arrays

\section{INTRODUCTION}

Solar Power Satellites (SPSs), or Space-Based Solar Power (SBSP) systems are used to collect the solar power and convert it into microwave energy to be beamed to a ground station antenna on earth $[1,2]$. Several SPS proposals have been introduced since the first SPS concept, proposed by Peter Glaser in 1968. NASA with the US Department of Energy (DOE) presented the first SPS reference model in 1978. The second reference SPS model was introduced by the Japanese Aerospace eXploration Agency (JAXA) in 2000, featuring electronic beam steering instead of mechanical antenna rotation. Recently, NASA and JAXA introduced improved models, while other agencies in different countries became interested and proposed several SPS models to be launched in different orbits. The latest SPS concept is the smaller SPS constellations, devised to overcome challenges such as weight, cost, and launching problems. Overviews of the recent international SPS models and research are presented in [3-5]. The specifications of NASA and JAXA reference models are presented in Table I, as published in [6].

TABLE I. REFERENCE SPS MODELS' PARAMETERS

\begin{tabular}{|c|c|c|c|c|}
\hline Model & Old JAXA & JAXA_1 & JAXA_2 & NASA \\
\hline Frequency & $5.8 \mathrm{GHz}$ & $5.8 \mathrm{GHz}$ & $5.8 \mathrm{GHz}$ & $2.45 \mathrm{GHz}$ \\
\hline Transmitting antenna diameter & $2.6 \mathrm{Km}$ & $1 \mathrm{Km}$ & $1.93 \mathrm{Km}$ & $1 \mathrm{Km}$ \\
\hline Transmitted power & $1.3 \mathrm{GW}$ & $1.3 \mathrm{GW}$ & $1.3 \mathrm{GW}$ & $6.72 \mathrm{GW}$ \\
\hline
\end{tabular}

An SPS system consists of the space and the ground stations. The space station consists of a solar energy collector, a microwave generator, and a transmitting antenna array, while the ground station mainly consists of the receiving antenna (rectenna). The major SPS benefits are the permanent transfer of solar power from space to earth wirelessly, without cables, grids, towers, and carbon emissions. The main drawbacks of the SPS technology are interferences and biological effects associated with the wireless transmission of electricity using High Power Microwave (HPM) signals [7-9]. Efficiency improvement is one of the main challenges of SPS systems, as it can reduce cost, interferences, and biological effects. The SPS efficiency is a combination of DC-RF conversion efficiency, RF-DC conversion efficiency [10], and beam efficiency. Beam efficiency depends on the transmitting and receiving antenna specifications, the wavelength, and the separation distance between the space and ground stations [11].

To improve beam efficiency, the transmitting antenna should be designed to radiate a narrow and controllable beam with suppressed sidelobes to concentrate the power on the receiving antenna aperture. Therefore, a phased array antenna with retro-directive beam control is usually used for beaming high-power microwaves to the receiving antenna on earth [12- 
15]. Tapered excitation is also commonly used to suppress the sidelobes, and thence more concentrated microwave power will be collected by the receiving antenna aperture area. The most famous excitation tapering techniques are Chebyshev, Taylor, Villeneuve, Gaussian, and binomial distributions. The radiating elements of the phased antenna system might be dipole, microstrip patch, reflector, horn, or Slotted Waveguide Antenna (SWA).

Due to their planar and rigid construction with low loss, high power handling capability, and high efficiency, SWAAs are more attractive candidates for application in microwave systems such as wireless communications, radars, and SPS systems [16-21]. The general principles and components of microwave power transmission systems and their space applications are outlined in [16], while several SWA arrays were designed in [17] with different tapered distributions for high power microwave applications. A slotted waveguide linear antenna array with 16 elements was designed at $9.4 \mathrm{GHz}$ for radar applications and simulated using HFSS in [18]. Subarraying techniques were applied in [19] with conventional longitudinal slot arrays to increase the relative bandwidth up to $15 \%$ at X-band for SAR applications. A procedure based on Elliott's model was proposed in [20] to design shaped beam planar arrays. An analytical procedure was introduced in [21], based on Babinet's principle, to design and construct a slotted waveguide antenna array at $2.45 \mathrm{GHz}$.

Recently, trapezoidal-shaped slots were used for increasing the bandwidth of SWAAs on 5G applications [22]. Slots were cut on the surface of the circular waveguide WC69 and filled with Teflon. A $4.54 \%$ of fractional bandwidth of the $24 \mathrm{GHz}$ center frequency was achieved with gains up to $14.71 \mathrm{dBi}$ over the operating bandwidth. A planar resonant SWAA of $24 \times 24$ longitudinal slots on the broad surface of a rectangular waveguide was designed and simulated for satellite communications within the $29.5-31 \mathrm{GHz}$ frequency band in [23], using the sub arraying technique, but without achieving the required 5\% fractional bandwidth. Moreover, the simulated SLL was found to be higher than the predicted $20 \mathrm{~dB}$. The simulation results produced approximately the designed gain of $30 \mathrm{dBi}$ and $3^{\circ}$ beamwidth in both azimuth and elevation planes. An antenna with a metasurface placed inside at an optimized location was proposed in [24] for an efficient DC-RF SPS module at $2.45 \mathrm{GHz}$ center frequency, without stating any further design details or parameters. The presented antenna patterns showed that the H-plane beamwidth was wider than the E-plane. Simplified closed-form equations were used in [25] to calculate the slots' lengths, widths, and their distribution along the length of the radiating and feeder SWAs. This method was applied to design an $8 \times 8$ planar SWAA at $3.952 \mathrm{GHz}$ for maritime applications. The obtained HPBWs were $9.6^{\circ}$ and $11.4^{\circ}$ with SLRs of $22.3 \mathrm{~dB}$ and $28.1 \mathrm{~dB}$ for $\mathrm{E}$ and $\mathrm{H}$ planes, respectively.

Slots are ideal radiating elements that can be easily cut into the waveguide walls incorporating their waveguide feeding structures without the need for special matching networks. Such integrated and robust antenna structures are conducive for the space stations of SPS systems. The radiating slots may be cut on the waveguide walls in longitudinal (shunt), transversal (series), or inclined shapes. The slotted waveguide antenna arrays can be resonant (standing) or non-resonant (traveling) waves. The traveling wave array has larger bandwidth but lower efficiency due to the power absorbed by the terminating matched loads. Also, the main beam direction of the traveling wave SWAA depends on the operating frequency. On the other hand, resonant SWAA has a narrower beamwidth with higher efficiency, its main beam direction is frequency independent, and it is always perpendicular to its array axis (broadside). Due to these attractive features, this study adopted the resonant SWAA for SPS systems.

To the best of our knowledge, no published papers have yet discussed an SWAA design to meet the operational requirements of the reference SPS models, apart from the scientific reports of NASA [26, 27] and JAXA [28, 29]. Therefore, this study aims to analyze, design, and simulate operable SWA arrays for the NASA and JAXA SPS reference models using closed-form equation, Babinet's principle, and different software packages such as PCAAD, Antenna Magus, and CST Microwave Studio.

\section{SWAA PARAMETERS AND DESIGN PROCEDURES}

The main objective of this study was to design SWA arrays as radiating elements for SPS transmitting antenna systems. The designed SWA arrays were simulated, and their performance was evaluated on the operational requirements of NASA and JAXA SPS models shown in Table II.

TABLE II. TYPICAL TRANSMITTING ANTENNA PARAMETERS FOR NASA AND JAXA SPS MODELS

\begin{tabular}{|cc|c}
\hline Parameter & NASA Model & JAXA Model \\
\hline Frequency bandwidth [GHz] & $2.4-2.5$ & $5.8-5.9$ \\
\hline Operating frequency [GHz] & 2.45 & 5.8 \\
\hline HPBW beamwidth [degrees] & $<4^{\circ}$ & $<4^{\circ}$ \\
\hline Directivity [dBi] & $>30$ & $>30$ \\
\hline Antenna diameter & $1 \mathrm{Km}$ & $1 \mathrm{Km}$ \\
\hline Sidelobe level (SLL) [dB] & $<20$ & $<20$ \\
\hline Output power (beamed to earth) & $>1.3 \mathrm{GW}$ & $>1.3 \mathrm{GW}$ \\
\hline
\end{tabular}

The first step in designing an SWA array is to choose the proper waveguide based on the frequency band. Figure 1 shows the rectangular waveguide and its cross-section dimensions.

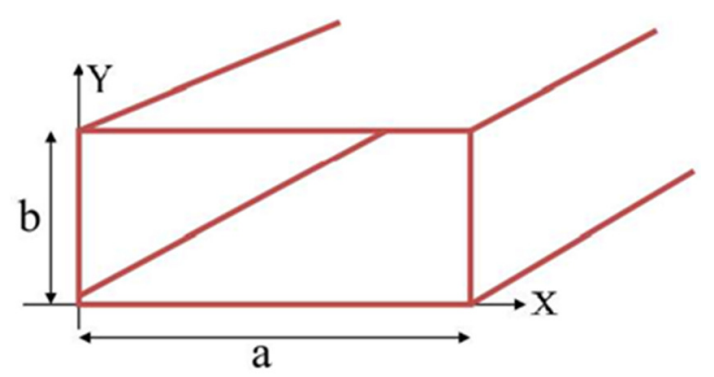

Fig. 1. Rectangular waveguide geometry and cross-sectional dimensions.

Assuming the dominant mode TE10 is propagating, the waveguide parameters are:

$$
\lambda_{c m}=\frac{2}{\sqrt{\left(\frac{m}{a}\right)^{2}+\left(\frac{n}{b}\right)^{2}}} ; \lambda_{g}=\frac{\lambda}{\sqrt{1-\lambda^{2} / \lambda_{c m}^{2}}}
$$




$$
\begin{gathered}
Z_{g T E}=\frac{\omega \mu}{\beta}=\eta \frac{\lambda_{g}}{\lambda}, \quad P=\frac{1}{4} \frac{\left|E_{m}\right|^{2}}{Z_{g T E 10}} \\
\alpha_{c}=\frac{P_{L}}{2 P}=\frac{1}{\sigma \delta b \eta} \frac{f_{c 10}}{f} \frac{\left[\left(\frac{f}{f_{c 10}}\right)^{2}+\left(\frac{2 b}{a}\right)\right]}{\sqrt{\left(f / f_{c 10}\right)^{2}-1}}\left[\frac{N e p}{m}\right], \delta=\sqrt{\frac{2}{\omega \mu \sigma}}, \eta=\sqrt{\frac{\mu}{\varepsilon}}
\end{gathered}
$$

where $\lambda_{c}$ is the cutoff wavelength, $\lambda_{g}$ is the guide wavelength, $Z_{g T E}$ is the guide impedance for TE mode, and $E_{m}$ and $P$ are the electric field amplitude and power propagating into the waveguide, respectively. The attenuation constant due to power losses $\left(\mathrm{P}_{\mathrm{L}}\right)$ in the waveguide conducting walls is $\alpha_{c}$. Based on the frequency bands of both NASA and JAXA SPS models, the appropriate rectangular waveguides were chosen, their parameters were calculated using the above equations, and the results are presented in Table III.

TABLE III. WAVEGUIDES PARAMETERS FOR SPS MODELS

\begin{tabular}{|c|c|c|}
\hline Parameter & $\begin{array}{c}\text { NASA } \\
\text { Model }\end{array}$ & $\begin{array}{c}\text { JAXA } \\
\text { Model }\end{array}$ \\
\hline SPS frequency range $[\mathrm{GHz}]$ & $2.4-2.5$ & $5.8-5.9$ \\
\hline Operating frequency $[\mathrm{GHz}]$ & 2.45 & 5.8 \\
\hline Free space wavelength $(\lambda)[\mathrm{mm}]$ & 122.45 & 51.72 \\
\hline Proper rectangular waveguide & WR340 & WR159 \\
\hline Waveguide frequency range $[\mathrm{GHz}]$ & $2.2-3.3$ & $4.9-7.05$ \\
\hline Waveguide dimensions $[\mathrm{mm}]$ & $a=86.36$, & $a=40.39$, \\
& $b=43.18$ & $b=20.19$ \\
\hline Cutoff wavelength $\left(\lambda_{c}\right)$ for TE10 $[\mathrm{mm}]$ & 172.72 & 80.77 \\
\hline Cutoff frequency $\left(f_{c}\right)$ for TE10 $[\mathrm{GHz}]$ & 1.737 & 3.714 \\
\hline Guide wavelength $\left(\lambda_{g}\right)[\mathrm{mm}]$ & 173.62 & 67.34 \\
\hline Propagation constant $(\beta)$ for TE10 $[\mathrm{rad} / \mathrm{m}]$ & 36.206 & 93.323 \\
\hline Attenuation coefficient $\left(\alpha_{c}\right)[\mathrm{dB} / \mathrm{m}]$ & 0.01 & 0.04 \\
\hline Waveguide impedance $(\mathrm{ZgTE} 10)[\Omega]$ & 534.5 & 490.8 \\
\hline
\end{tabular}

Figure 2 represents the sketch of a linear resonant SWAA of $\mathrm{N}$ slots (a) and its equivalent circuit (b). The slots are cut longitudinally on the rectangular waveguide broad wall. The waveguide ends are terminated with short circuits, a quarter guide wavelength $\left(\lambda_{g} / 4\right)$ apart from the centers of last slots. The slot elements' centers are placed half guide wavelength $\left(d_{s}=\lambda_{g} / 2\right)$ apart and are offset on the opposite side of the waveguide centerline to ensure uniform phase excitation. The slot length was chosen to be resonant, approximately equal to half wavelength $(L s=\lambda / 2)$, and thus the longitudinal slot was equivalent to a shunt conductance $\mathrm{g}$, as shown in Figure 2(b).
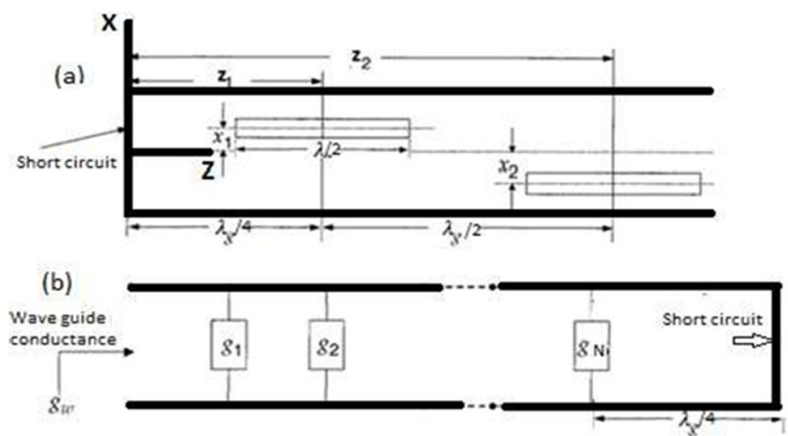

Fig. 2. (a) Resonant shunt slotted waveguide array and (b) its equivalent circuit.

An equation was developed in [30] for calculating the normalized slot conductance $\left(g_{n}=G_{n} / g_{w}\right)$ of the $n$-th narrow slot radiating into a half-space, which accounts for the actual slot length. This can be used to calculate each slot displacement $x_{n}$, $n=1,2, \ldots N$ :

$$
g_{n}=2.09 \frac{\lambda_{g}}{\lambda_{0}} \frac{a}{b}\left(\cos \left(\frac{0.464 \pi \lambda_{0}}{\lambda_{g}}\right)-\cos (0.464 \pi)\right)^{2} \sin ^{2}\left(\frac{\pi x_{n}}{a}\right)
$$

For perfect matching, the waveguide normalized input conductance should be equal to the total equivalent normalized conductance of the shunt slots for the end-fed linear SWA array, i.e.:

$$
\sum_{n=1}^{N} g_{n}=1
$$

The slot conductance is proportional to its radiated power, according to:

$$
g_{n}=C a_{n}^{2}
$$

where $a_{n}$ is the excitation coefficient of the $n$-th slot. The excitation coefficients are adjusted to yield the required pattern. Equation (6) was substituted into (5) to get the constant $C$. Then substituting $C$ into (6) to get the normalized $n$-th slot's conductance $g_{n}$, and thence the $n$-th slot offset $x_{n}$ can be calculated from (4). For a center-fed linear SWA array terminated by short circuits at both ends, the matching condition provided by (5) will be:

$$
\sum_{n=1}^{N} g_{n}=2
$$

For the end-fed linear uniform array of $\mathrm{N}$ identical shunt slots, the normalized slot conductance is simply computed from (5) to be $g_{n}=1 / N$ for all $n$. Accordingly, the offset $x_{n}$ will be the same. Alternatively, Babinet's principle could be used directly to design the SWA array in terms of its dual dipole array, where the (thin) slot admittance $Y_{s}$ is related to its complementary thin wire dipole impedance $Z_{w}$ by:

$$
Y_{s}=G_{s}+j B_{s}=4 Z_{w} / \eta^{2}
$$

where $\eta=\sqrt[2]{\frac{\mu}{\varepsilon}}$ is the intrinsic impedance of the surrounding medium. Substituting resonant conditions in free space $(\eta=377$, $Z_{w}=73.2$ ) into (8) gives the resonant slot conductance as:

$$
G_{S}=\frac{(4 \times 73.2)}{377^{2}}=2.06 \mathrm{mS}
$$

The power radiated by a slot is $P_{s}=V_{s}^{2} G_{s}$. Assuming a uniform electric field distribution across the slot aperture, $E s(\mathrm{x}, \mathrm{z})=E s$, and slot voltage $V_{s}=E_{s} W_{s}$, where $W_{s}$ is the slot width. The minimum slot width $W_{s}^{\text {min }}$ is determined from the maximum power handling capability of the slot $\left(P^{\max }\right)$, which corresponds to applying the breakdown electric field $E_{b d}$. Substituting by the slot conductance $G_{s}$ from (9), and the free space breakdown electric field $E_{b d}=3 \mathrm{MV} / \mathrm{m}=E s$, the minimum slot width will be equal to:

$$
W_{s}^{\min }=\left(\frac{1}{E_{b d \sqrt{G s}}} \sqrt{P^{\max }}\right)=0.00735 \sqrt{P^{\max }}
$$

For a thin slot, the length to width slot ratio $\left(L_{s} / W_{s}\right)$ should be kept larger than 10 .

The required number of slots $N$ in the SWA array can be estimated based on the maximum power handling capability, 
specified voltage standing wave ratio VSWR, bandwidth, and the required beamwidth and directivity. The required transmitting antenna diameter is approximately $1 \mathrm{Km}$ for both NASA and JAXA models, as shown in Table II. The equivalent square aperture array shall have approximately equal $\mathrm{x}$ and $\mathrm{y}$ dimensions as $L_{x}=L_{y}=886.41 \mathrm{~m}$. For a center-fed linear array, its waveguide length is $L_{g}=\left(N_{s}-1\right) d+\lambda_{g} / 4+\lambda_{g} / 4$, where $d$ is the inter-element spacing from slots' centers equal to $g / 2$ for a resonant array. Thus, the number of slot elements in $\mathrm{x}$ or $\mathrm{y}$ dimensions is $N_{s}=N_{x}=N_{y}=1772.82 / \lambda_{g}$, and the total number of slots $N_{t}$ required to a $1 \mathrm{Km}$ diameter SPS transmitting antenna area with a planar square array of slotted waveguides is calculated as:

$$
N_{t}=\left(N_{s}\right)^{2}=\left(\frac{1772.82}{\lambda_{g}}\right)^{2}
$$

\section{SWA DESIGN AND SIMULATION}

Based on the previous design procedures, several linear SWA arrays having different numbers of slots with uniform and tapered distributions were designed and simulated using software packages such as PCAAD, Antenna Magus, and CST Microwave Studio. Linear uniform resonant SWA arrays with 10 elements were designed for $2.45 \mathrm{GHz}$ and $5.8 \mathrm{GHz}$ frequencies. Figure 3 shows the field pattern of its equivalent half-wave dipoles array for $2.45 \mathrm{GHz}$, where $3 \mathrm{~dB}$ beamwidth was $7.05^{\circ}$, directivity was $11.6 \mathrm{~dB}$, and SLL was $-13 \mathrm{~dB}$. Chebyshev excitations were used for the same array to get its field pattern, where the $3 \mathrm{~dB}$ beamwidth was $7.790^{\circ}$, which was slightly wider than its uniform excitation counterpart, but the SLL was largely suppressed to -20dB. Similar patterns with approximately the same pattern parameters were obtained for the arrays designed for $5.8 \mathrm{GHz}$.

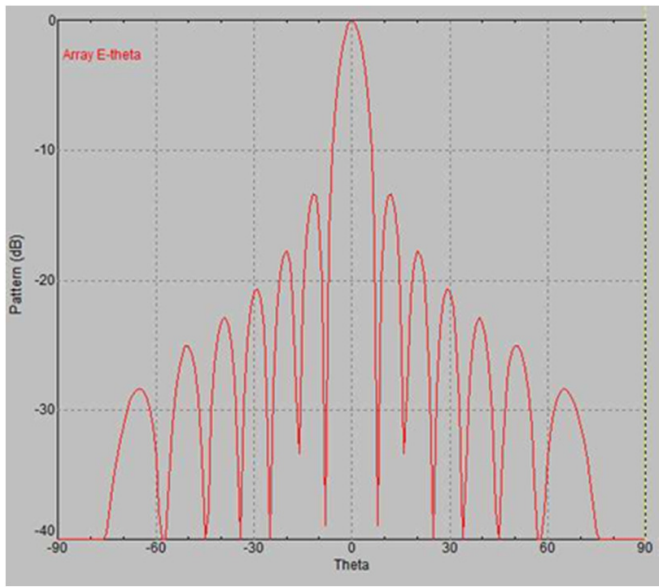

Fig. 3. The field patterns of a linear uniform array having10 uniform excitation half-wave dipoles at $2.45 \mathrm{GHz}$.

Other linear SWA arrays, having 20 elements, were designed for $2.45 \mathrm{GHz}$ and $5.8 \mathrm{GHz}$, and Figure 4 shows the field patterns for $5.8 \mathrm{GHz}$. HPBW was narrower and decreased by more than a factor of half the 10-element arrays for both uniform and Chebyshev excitations. Similar patterns were obtained for the designed array at $2.45 \mathrm{GHz}$.

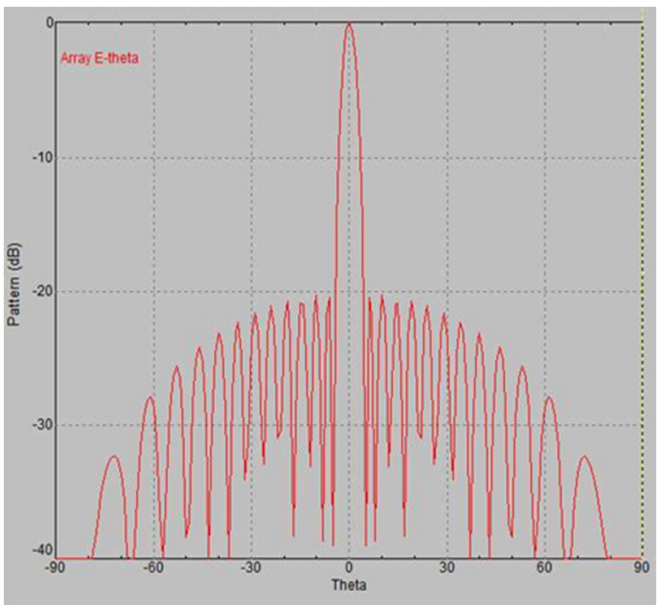

Fig. 4. The field patterns of linear uniform array of 20 Chebyshev excitation half wave dipoles at $5.8 \mathrm{GHz}$.

Planar arrays of $20 \times 20$ equivalent half-wave dipoles with the same dual-slot dimensions $\left(L_{s}=\lambda / 2, d_{x}=g / 2\right.$, and $d_{y}=$ waveguide width $a$ ) were designed for $2.45 \mathrm{GHz}$ and $5.8 \mathrm{GHz}$. The obtained patterns for both frequencies were quite similar. Figures 5(a) and 5(b) show the array patterns with uniform and Chebyshev excitations at $2.45 \mathrm{GHz}$, where a pencil beam was obtained with $3^{\circ} \mathrm{HPBW}$ in both planes.

(a)

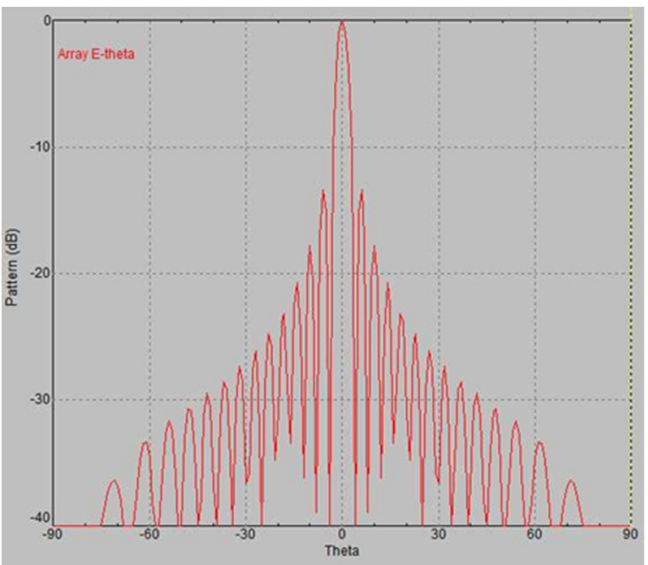

(b)

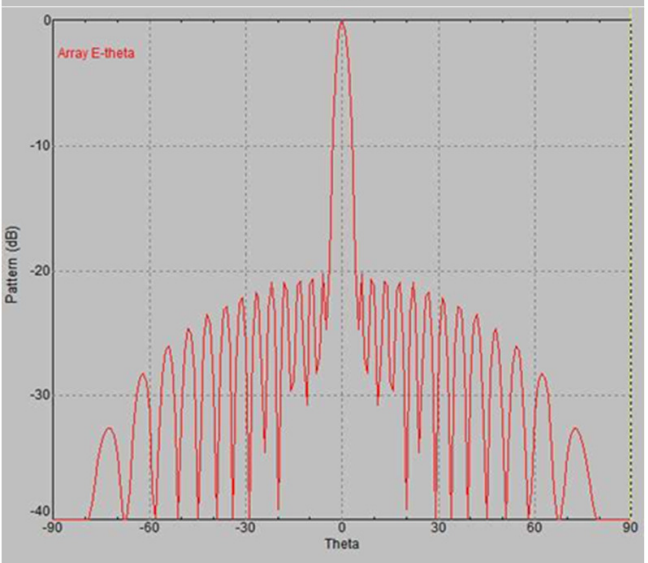

Fig. 5. The field patterns for a planar uniform array of $20 \times 20$ half-wave dipoles at $2.45 \mathrm{GHz}$. (a) Uniform and (b) Chebyshev excitation. 
Linear and planar resonant SWA arrays having 10 and 20 slots with uniform and Villeneuve distributions were simulated for $2.45 \mathrm{GHz}$ and $5.8 \mathrm{GHz}$.

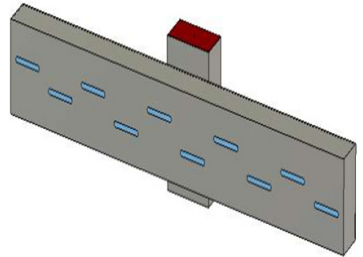

(a)

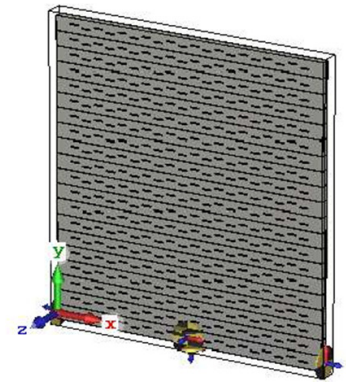

(b)
Fig. 6. Simulated resonant SWA arrays. (a) Linear center-fed array, (b) end-fed planar array.

The simulated SWA arrays are shown in Figures 6(a) and (b) for linear and planar structures respectively. The SWA arrays' parameters were obtained by plugging the data of Table II into (4)-(11). Table IV shows the results for the 10-element linear SWAAs on $2.45 \mathrm{GHz}$ and $5.8 \mathrm{GHz}$.

TABLE IV. DESIGN PARAMETERS OF LINEAR 10-ELEMENT UNIFORM RESONANT SWA ARRAYS FOR SPS MODELS

\begin{tabular}{|c|c|c|}
\hline Parameter & NASA Model & JAXA Model \\
\hline No. of slot elements & 10 & 10 \\
\hline Slot length $\left(L_{s}\right)[\mathrm{mm}]$ & 61.22 & 25.86 \\
\hline Slot spacing $\left(d_{s}\right)[\mathrm{mm}]$ & 86.81 & 33.67 \\
\hline Slot width $\left(W_{s}\right)[\mathrm{mm}]$ & 6 & 2.8 \\
\hline Slot offset $\left(x_{s}\right)[\mathrm{mm}]$ & 8.85 & 5.41 \\
\hline Ratio $\left(L_{s} W_{s}\right)$ & 10.2 & 9.24 \\
\hline Short circuit end spacing & 43.41 & 16.84 \\
\hline $\begin{array}{c}\text { Distance from feed slot to } \\
\text { feed termination }[\mathrm{mm}]\end{array}$ & 79.36 & 33.52 \\
\hline Feed waveguide length $[\mathrm{mm}]$ & 158.7 & 67.05 \\
\hline Feed slot length $[\mathrm{mm}]$ & 57.9 & 24.46 \\
\hline Feed slot width & 6.118 & 2.584 \\
\hline Feed slot angle $[$ degrees] & 45 & 45 \\
\hline SWAA Z-dimension $[\mathrm{mm}]$ & 793.6 & 335.2 \\
\hline SWAA X-dimension $[\mathrm{mm}]$ & 158.7 & 67.05 \\
\hline SWAA Y-dimension $[\mathrm{mm}]$ & 96.06 & 40.52 \\
\hline
\end{tabular}

Gain (Total)

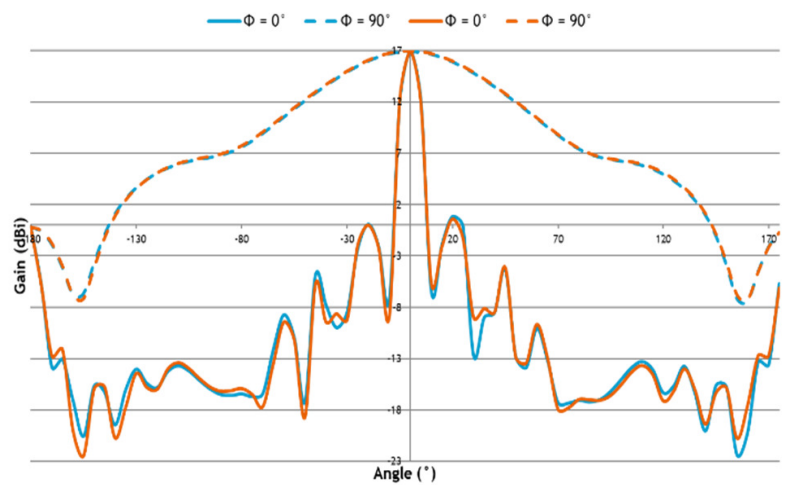

Fig. 7. Radiation patterns of linear resonant center-fed 10-element uniform SWA arrays at $2.45 \mathrm{GHz}$ (blue) and $5.8 \mathrm{GHz}$ (red).
The optimized array patterns for SWA arrays with Villeneuve distributions are shown in Figure 7. The blue line refers to $2.45 \mathrm{GHz}$ and the red to $5.8 \mathrm{GHz}$. Their reflection coefficients (S11) are plotted versus frequency in Figure 8. Similarly, more linear resonant 10 - and 20-slot element SWAAs with uniform and Villeneuve distributions were designed and simulated. Such arrays were designed to be used as subarrays for the required planar SWAA arrays by NASA and JAXA SPS models. The radiation patterns of a 20 -slot linear uniform array with uniform and Villeneuve distributions are shown in Figure 9.



Fig. 8. Reflection coefficients (S11) of linear resonant center-fed 10elements uniform SWA arrays for NASA (blue) and JAXA (red) SPS models.

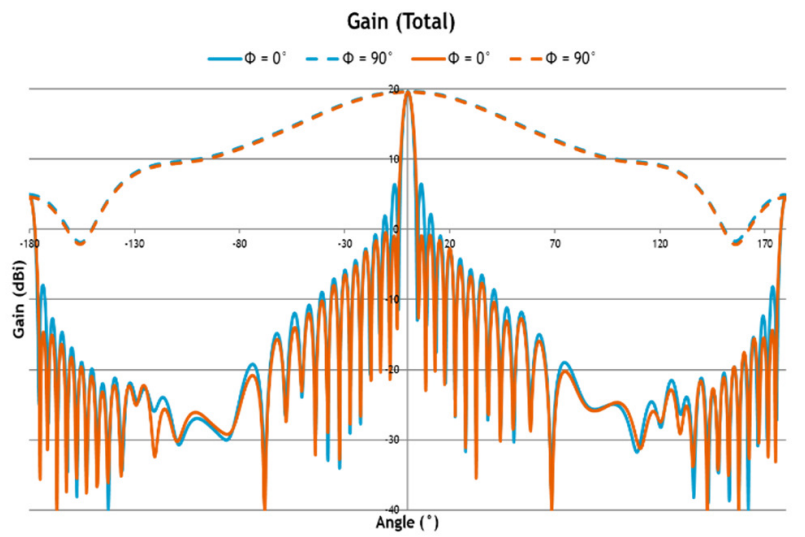

Fig. 9. Radiation patterns of linear resonant 20-element uniform SWA arrays for uniform (blue) and Villeneuve (red) excitations.

\section{SWA PERFORMANCE EVALUATION}

The parameters of the previously described designs are summarized and presented in Tables V-VII. Tables V and VI show the pattern parameters for the slot complementary (dipole) linear and planar arrays of 10 and 20 element with uniform and Chebyshev distributions for both models. Table VII presents the antenna parameters of the linear SWA arrays with uniform and Villeneuve excitations. Studying and comparing the antenna parameters for the uniform SWA arrays concludes to:

- The HPBW and SLL of linear uniform arrays are approximately the same in Tables V and VI, and are very 
close to those produced by the simulation of actual SWA arrays (Table VII).

- The pattern parameters for linear uniform arrays in Tables V and VII with tapered distributions are approximately the same for both Chebyshev (Table V) and Villeneuve (Table VII).

- The calculated pattern parameters, specifically the directivity Do (maximum directive gain) and the $3 \mathrm{~dB}$ HPBW in Table V, are compatible with the classical array theory, which states that for a linear uniform broadside array, if $N_{d}>>\lambda$, then, $D o=2 N_{d} / \lambda$, and $H P B W=101.5 \lambda / N_{d}$.

- The obtained relative bandwidth, shown in Table VII, is decreased to half when the number of elements is doubled from 10 to 20 for both uniform and Villeneuve distributions, while the HPBW is getting narrower and halved when the number of elements is doubled.

- The planar SWA arrays presented in Table VI are exhibiting narrow beams in both principal planes and thus the overall directivity was increased and found to be slightly larger than the summation of directivities of their constituting linear arrays in $\mathrm{dB}$ scale, which is again compatible with the classical array theory.

- The bandwidth of all designed arrays in Table VII satisfied the bandwidth operational requirements for both SPS models $(\mathrm{BW}=100 \mathrm{MHz})$, except the 20 element arrays at $2.45 \mathrm{GHz}$, where the bandwidth was found to be approximately equal to $50 \mathrm{MHz}$ for both uniform and Villeneuve distributions.

TABLE V. LINEAR SLOT COMPLEMENTARY (DIPOLE) ARRAYS' SPECIFICATIONS

\begin{tabular}{|c|c|c|c|c|c|c|c|c}
\hline \multirow{2}{*}{ Pattern Parameters } & \multicolumn{3}{|c|}{ NASA Model } & \multicolumn{3}{c}{ JAXA Model } \\
\cline { 2 - 8 } & \multicolumn{2}{|c|}{$\mathbf{N = 1 0}$} & \multicolumn{2}{|c|}{$\mathbf{N = 2 0}$} & \multicolumn{2}{c}{ N=10 } & \multicolumn{2}{c}{ N=20 } \\
\cline { 2 - 8 } & Uniform & Chebyshev & Uniform & Chebyshev & Uniform & Chebyshev & Uniform & Chebyshev \\
\hline Half power beamwidth (HPBW) $[\mathrm{deg}]$ & 7.05 & 7.79 & 3.42 & 3.68 & 7.76 & 8.40 & 3.8 & 4.05 \\
\hline Sidelobelevel (SLL) [dB] & -13.3 & -20.00 & -13.4 & -20.00 & -13.14 & -20.00 & -13.28 & -20.00 \\
\hline Directivity (Do) $[\mathrm{dB}]$ & 11.6 & 11.4 & 14.6 & 14.4 & 11.3 & 11.1 & 14.2 & 14.1 \\
\hline
\end{tabular}

TABLE VI. PLANAR SLOT COMPLEMENTARY (DIPOLE) ARRAYS' SPECIFICATIONS

\begin{tabular}{|c|c|c|c|c|c|c|c|c|}
\hline \multirow{3}{*}{ Parameters } & \multicolumn{4}{|c|}{ NASA Model } & \multicolumn{4}{|c|}{ JAXA Model } \\
\hline & \multicolumn{2}{|c|}{$10 \times 10$} & \multicolumn{2}{|c|}{$20 \times 20$} & \multicolumn{2}{|c|}{$10 \times 10$} & \multicolumn{2}{|c|}{$20 \times 20$} \\
\hline & Uniform & Chebyshev & Uniform & Chebyshev & Uniform & Chebyshev & Uniform & Chebyshev \\
\hline Half power beamwidth (HPBW) [deg] & $7.05 \times 6.02$ & $7.79 \times 7.50$ & $3.42 \times 3.01$ & $3.68 \times 3.52$ & $7.67 \times 6.34$ & $8.4 \times 6.92$ & $3.80 \times 2.99$ & $4.05 \times 3.20$ \\
\hline Sidelobe level (SLL) [dB] & -13.01 & -20.30 & -13.25 & -20.31 & -13.30 & -20.40 & -13.40 & -20.33 \\
\hline Directivity (Do) $[\mathrm{dB}]$ & 24.8 & 24.6 & 31 & 30.8 & 24.70 & 24.5 & 30.90 & 30.7 \\
\hline
\end{tabular}

TABLE VII. SWAA SPECIFICATIONS

\begin{tabular}{|c|c|c|c|c|c|c|c|c|}
\hline \multirow{3}{*}{ Specification } & \multicolumn{4}{|c|}{ NASA Model } & \multicolumn{4}{|c|}{ JAXA Model } \\
\hline & \multicolumn{2}{|c|}{$N=10$} & \multicolumn{2}{|c|}{$\mathbf{N}=\mathbf{2 0}$} & \multicolumn{2}{|c|}{$\mathbf{N}=\mathbf{1 0}$} & \multicolumn{2}{|c|}{$\mathrm{N}=\mathbf{2 0}$} \\
\hline & Uniform & Villeneuve & Uniform & Villeneuve & Uniform & Villeneuve & Uniform & Villeneuve \\
\hline Half power beamwidth (HPBW) [deg] & 7.75 & 8.51 & 3.87 & 4.21 & 7.76 & 8.49 & 3.87 & 4.21 \\
\hline Sidelobe level (SLL) $[\mathrm{dB}]$ & -13.01 & -20.61 & -13.25 & -20.51 & -13.14 & -20.40 & -13.28 & -20.37 \\
\hline Directivity (Do) [dB] & 16.78 & 16.62 & 19.70 & 19.60 & 16.77 & 16.62 & 19.73 & 19.59 \\
\hline Bandwidth (below the $-10 \mathrm{~dB}$ level) $[\mathrm{MHz}]$ & 104.1 & 97.20 & 50.32 & 47.34 & 246.4 & 230.1 & 119.1 & 112.1 \\
\hline Relative bandwidth (\%) & 4.25 & 3.97 & 2.05 & 1.93 & 4.25 & 3.97 & 2.05 & 1.93 \\
\hline
\end{tabular}

Practically, weight, dimensions, and gain are more critical than the bandwidth for SPS operations. Therefore, this study recommends the 20-element linear uniform SWAA to be the subarray of the planar square array for SPS transmitting antennas. Equation (11) can be used to calculate the total number of slots $N_{t}$ on the proposed planar square arrays for both SPS models and the total number of $20 \times 20$ planar array modules required to cover the whole transmitting antenna area, whose diameter is $1 \mathrm{Km}$ for both SPS models. These calculations show that the required number of $20 \times 20$ planar array modules is approximately 260,658 modules for the NASA and 1,732,699 modules for the JAXA models. Accordingly, and due to the classical array theory calculations, the $3 \mathrm{~dB}$ beamwidth (HPBW) was found to be approximately $0.007^{\circ}$ and $0.003^{\circ}$ for the transmitting antennas of NASA and JAXA SPS models, respectively, and the overall directivity (Do) was found to be approximately equal to $83.22 \mathrm{~dB}$ and $90.7 \mathrm{~dB}$ for the NASA and JAXA SPS models, respectively.

\section{CONCLUSION}

Based on the SPS operational requirements, several linear and planar uniform SWA arrays with uniform and tapered excitation distributions were designed and simulated using different software packages. The antenna patterns and parameters of the designed SWA arrays obtained from the slot complementary (dipoles) elements, based on Babinet's principle, were found to be quite similar to those obtained by the simulation of actual SWA arrays having the same parameters. Based on extensive calculations, a planar array of $20 \times 20$ slot elements was proposed to be a module for the completely planar SWA array to cover the $1 \mathrm{Km}$ diameter area of the transmitting antennas for both NASA and JAXA SPS models. The proposed transmitting antennas' parameters were found to fulfill the operational requirements for both SPS models. However, the antenna specifications of the proposed planar SWA array for the JAXA are better than for the NASA SPS model. Such transmitting antennas achieve pencil beam with very narrow HPBW in the principle planes, which is 
approximately $0.007^{\circ}$ and $0.003^{\circ}$ for NASA and JAXA SPS models, respectively. Moreover, the achieved overall directivity was found to be approximately $83.22 \mathrm{~dB}$, and $90.7 \mathrm{~dB}$ for NASA and JAXA SPS models, respectively. Experimental verification is planned for the future. Due to the higher operating frequency, the results confirmed that the antenna specifications of the proposed planar SWA array for the JAXA were better than for the NASA SPS model. Several SPS geometrical and technical challenges such as size, weight, cost, assembly, and launching are still under investigation. Smaller satellite constellations are some latest proposals to overcome some of these challenges.

\section{REFERENCES}

[1] J. O. McSpadden and J. C. Mankins, "Space solar power programs and microwave wireless power transmission technology," IEEE Microwave Magazine, vol. 3, no. 4, pp. 46-57, Dec. 2002, https://doi.org/10.1109/ MMW.2002.1145675.

[2] D. M. Flournoy, Solar Power Satellites, 2nd edition. New York: Springer, 2011.

[3] J. D. Dakora, I. E. Davidson, and G. Sharma, "Review of Modern Solar Power Satellite and Space Rectenna Systems," in 2020 International Conference on Artificial Intelligence, Big Data, Computing and Data Communication Systems (icABCD), Aug. 2020, pp. 1-5, https://doi.org/10.1109/icABCD49160.2020.9183884.

[4] S. DebBarman, S. Gupta, and O. P. Das, "A Review: Space Based Solar Power (Sbsp) in Development of Smart City," Social Science Research Network, Rochester, NY, Jun. 2020, Art. no. 3621435. https://doi.org/ $10.2139 / \mathrm{ssrn} .3621435$.

[5] J. A. Vedda and K. L. Jones, "Space-based Solar Power: A near-term Investment Decision," Center for Space Policy and Strategy, Oct. 2020.

[6] "URSI White Paper on Solar Power Satellite (SPS) Systems and Report of the URSI Inter-Commission Working Group on SPS," URSI Inter-commission Working Group on SPS.

[7] T. Hatsuda, K. Ueno, and M. Inoue, "Solar power satellite interference assessment," IEEE Microwave Magazine, vol. 3, no. 4, pp. 65-70, Dec. 2002, https://doi.org/10.1109/MMW.2002.1145677.

[8] M. E. Bendib and A. Mekias, "Solar Panel and Wireless Power Transmission System as a Smart Grid for Electric Vehicles," Engineering, Technology \& Applied Science Research, vol. 10, no. 3, pp. 5683-5688, Jun. 2020, https://doi.org/10.48084/etasr.3473.

[9] H. Matsumoto, "Numerical estimation of SPS microwave impact on ionospheric environment," Acta Astronautica, vol. 9, no. 8, pp. 493-497, Aug. 1982, https://doi.org/10.1016/0094-5765(82)90095-9.

[10] J. O. McSpadden, L. Fan, and K. Chang, "A high conversion efficiency $5.8 \mathrm{GHz}$ rectenna," in 1997 IEEE MTT-S International Microwave Symposium Digest, Jun. 1997, vol. 2, pp. 547-550 vol.2, https://doi.org/ 10.1109/MWSYM.1997.602852.

[11] R. B. Vaganov, "Maximum power transmission between two apertures with the help of a wave beam," Journal of communications technology \& electronics, vol. 42, no. 4, pp. 397-402, 1997.

[12] N. Shinohara, J. Fujiwara, and H. Matsumoto, "Development of Active Phased Array with Phase-controlled Magnetrons," in Proceedings of ISAP2000, Fukuoka, Japan.

[13] M. C. Hatfield and J. G. Hawkins, "Design of an electronically-steerable phased array for wireless power transmission using a magnetron directional amplifier," in 1999 IEEE MTT-S International Microwave Symposium Digest (Cat. No.99CH36282), Jun. 1999, vol. 1, pp. 341344, https://doi.org/10.1109/MWSYM.1999.779489.

[14] M. M. Nahas and M. Nahas, "Bandwidth and Efficiency Enhancement of Rectangular Patch Antenna for SHF Applications," Engineering, Technology \& Applied Science Research, vol. 9, no. 6, pp. 4962-4967, Dec. 2019, https://doi.org/10.48084/etasr.3014.

[15] K. Hashimoto, K. Tsutsumi, H. Matsumoto, and N. Shinohara, "Space solar power system beam control with spread-spectrum pilot signals,"
URSI Radio Science Bulletin, vol. 2004, no. 311, pp. 31-37, Dec. 2004, https://doi.org/10.23919/URSIRSB.2004.7909631.

[16] W. C. Brown and E. E. Eves, "Beamed microwave power transmission and its application to space," IEEE Transactions on Microwave Theory and Techniques, vol. 40, no. 6, pp. 1239-1250, Jun. 1992, https://doi.org/10.1109/22.141357.

[17] H. M. E. Misilmani, M. Al-Husseini, and and M. Mervat, "Design of Slotted Waveguide Antennas with Low Sidelobes for High Power Microwave Applications," Progress In Electromagnetics Research C, vol. 56, pp. 15-28, 2015, https://doi.org/10.2528/PIERC14121903.

[18] S. Murugaveni and T. Karthick, "Design of Slotted Waveguide Antenna for Radar Applications at X-Band," International Journal of Engineering Research \& Technology, vol. 3, no. 11, Nov. 2014.

[19] S. Sekretarov and D. M. Vavriv, "A Wideband Slotted Waveguide Antenna Array for SAR Systems," Progress In Electromagnetics Research $M$, vol. 11, pp. 165-176, 2010, https://doi.org/10.2528/ PIERM10010606.

[20] G. A. Casula, G. Mazzarella, and G. Montisci, "Design of Shaped Beam Planar Arrays of Waveguide Longitudinal Slots," International Journal of Antennas and Propagation, vol. 2013, Feb. 2013, Art. no. e767342, https://doi.org/10.1155/2013/767342.

[21] A. A. Eyadeh and M. N. Al-Ta'ani, "Performance Study of Wireless Systems with Switch and Stay Combining Diversity over $\alpha-\eta-\mu$ Fading Channels," Engineering, Technology \& Applied Science Research, vol. 9, no. 6, pp. 5047-5055, Dec. 2019, https://doi.org/10.48084/etasr.3100.

[22] H. R. D. Filgueiras, J. R. Kelly, P. Xiao, I. F. da Costa, and A. Cerqueira Sodré, "Wideband Omnidirectional Slotted-Waveguide Antenna Array Based on Trapezoidal Slots," International Journal of Antennas and Propagation, vol. 2019, Oct. 2019, Art. no. e3792980, https://doi.org/10.1155/2019/3792980.

[23] L. Ripoll and L. Valdez, "Design and Simulation of a Slot Waveguide Array Antenna (SWAA) for Satellite Communications," IOP Conference Series: Materials Science and Engineering, vol. 519, no. 1, May 2019, Art. no. 012035, https://doi.org/10.1088/1757-899X/519/1/012035.

[24] A. K. Baghel, S. S. Kulkarni, and S. K. Nayak, "Efficient Modeling of DC-RF module of Space Solar Power Satellite with Improved Antenna design and Metasurface," in 2019 IEEE International Conference on Microwaves, Antennas, Communications and Electronic Systems (COMCAS), Nov. 2019, pp. 1-4, https://doi.org/10.1109/ COMCAS44984.2019.8958029.

[25] H. M. El Misilmani, M. Al-Husseini, and K. Y. Kabalan, "Design procedure for planar slotted waveguide antenna arrays with controllable sidelobe level ratio for high power microwave applications," Engineering Reports, vol. 2, no. 10, 2020, Art. no. e12255, https://doi.org/10.1002/eng2.12255.

[26] H. Benson and M. Jenkins, "Satellite Power System Concept Development and Evaluation Program Volume VI Construction and Operations," National Aeronautics and Space Administration (NASA), Technical Memorandum 58233, 1981.

[27] R. H. Dietz, G. D. Arndt, J. W. Seyl, L. Leopold, and J. S. Kelley, "Satellite power system: Concept development and evaluation program. Volume 3: Power transmission and reception. Technical summary and assessment," National Aeronautics and Space Administration (NASA), Technical Memorandum 58238, Jul. 1981.

[28] H. Matsumoto, "Research on Solar Power Station and Microwave Power Transmission in Japan: Technology and Strategy (invited)," presented at the 2001 Asia-Pacific Radio Science Conference (AP-RASC '01), Tokyo, Japan, Jan. 2001.

[29] "Outline of the Basic Plan on Space Policy (Provisional Translation)," National Space Policy Secretariat, Cabinet Office, Japan, Jun. 2020.

[30] R. Elliott, "An improved design procedure for small arrays of shunt slots," IEEE Transactions on Antennas and Propagation, vol. 31, no. 1, pp. 48-53, Jan. 1983, https://doi.org/10.1109/TAP.1983.1143002. 